\title{
Haematuria of unknown origin
}

\author{
PATRICK H. O'ReILly \\ M.B., CH.B., L.R.C.P., M.R.C.S. \\ Department of Urology, University Hospital of South Manchester
}

\begin{abstract}
Summary
Sixty-four cases of haematuria of unknown origin are reviewed. The results are compared with findings from other centres and discussion follows regarding the management of these patients.
\end{abstract}

\section{Introduction}

The majority of patients presenting to any Urological centre complaining of haematuria will not present too much difficulty in diagnosis, and most will be found to be due to such conditions as urinary tract tumour, calculous disease, prostatic enlargement and urinary tract inflammation or trauma; others will fall into less common categories. However, any centre will have a further group of patients in whom, after the initial investigations and tests are completed, no cause is found, and these will present the clinician with a certain amount of consternation. In most centres this last group has been calculated to be between 5 and $10 \%$ of all haematuria cases. In a survey by Burkholder and colleagues (1969) at the Brooke General Hospital, Texas, twenty-five patients out of 237 fell into the unknown origin group-to their surprise the second largest group in their series. In the present series, sixty-four patients were entered in the Urology Disease Index at the University Hospital of South Manchester (UHSM), under the category under review, between January 1967 and July 1972. The majority of patients were referred to the unit by their GPs; some were referred by other consulting clinicians and some presented via the Casualty Department. Many had associated features which suggested possible causes for the bleeding, but no firm diagnosis on pathological grounds could be made. Although laboratory tests performed were somewhat variable, all but two patients had intravenous pyelograms and all but two had cystoscopies. Twelve had retrograde pyelograms and three renal angiograms. All were followed up as outpatients following their initial investigation; in three cases definite disease states later manifested themselves-the remainder remain an unsolved problem.

Correspondence: University Hospital of South Manchester, Nell Lane, West Didsbury, Manchester M20 8LR.

\section{Results}

Of the sixty-four cases reviewed, thirty-eight were men and twenty-six women. The average age in both groups was 50 years. During investigation, twentynine of the patients were found to have red blood cells in their urine. Thirty-nine of the patients had circumstantial features which may have contributed to the bleeding, but they were merely circumstantial and by no means conclusive. These findings are summarized in Table 1.

TABLE 1. Patients entered in UHSM urology disease index under haematuria of unknown origin

\begin{tabular}{|c|c|c|c|}
\hline Cases reviewed: & $\begin{array}{c}\text { Men } \\
38\end{array}$ & $\begin{array}{c}\text { Women } \\
26\end{array}$ & Tota \\
\hline Age (in years): & $\begin{array}{c}12-70 \\
\text { (average 50) }\end{array}$ & $\begin{array}{c}16-76 \\
\text { (average 50) }\end{array}$ & \\
\hline \multicolumn{4}{|l|}{ Associated features } \\
\hline Anticoagulants & 2 & 1 & 3 \\
\hline Hypertension & 8 & 4 & 12 \\
\hline Symptoms of prostatism & 6 & & 6 \\
\hline Symptoms of urinary & 10 & 4 & 14 \\
\hline Post intercourse & $\begin{array}{r}10 \\
1\end{array}$ & & $\begin{array}{r}14 \\
1\end{array}$ \\
\hline Post heavy drinking & 1 & & 1 \\
\hline Post athletic exercise & 2 & & 2 \\
\hline No associated features & 7 & 15 & 22 \\
\hline \multicolumn{4}{|l|}{ Subsequent diagnosis } \\
\hline Hypernephroma & 1 & & 1 \\
\hline Carcinoma renal pelvis & & 1 & 1 \\
\hline Sickle cell trait & & 1 & 1 \\
\hline Total & 38 & 26 & 64 \\
\hline
\end{tabular}

(1) Three patients were on anticoagulants at the time of their haematuria. This, of course, should not prevent full urological investigation-a number of our patients with bladder tumours have presented with haematuria while on anticoagulant drugs-for these drugs are not necessarily a cause of haematuria but may serve to bring other pathology to light.

(2) Twelve patients were found to be hypertensive, with diastolic pressures consistently above 110 and some as high as 150 .

(3) Fourteen patients had symptoms of urinary tract infection, such as frequency and urgency of 
micturition and dysuria, before or during the attack of bleeding, but at no time was any organism grown on culture or a significant number of cells found on microscopy of the urine.

(4) Six patients had symptoms and clinical evidence of prostatic enlargement.

(5) One case occurred after sexual intercourse.

(6) One case followed a heavy drinking bout.

(7) Two cases occurred only after heavy exercise.

(8) One patient in the group was eventually found to have a hypernephroma. This man presented to the clinic in 1966 with painless haematuria, confirmed on macroscopic and microscopic examination of the urine. All biochemical tests were within normal limits, as were intravenous pyelogram, cystoscopy and retrograde pyelogram. He was followed up in the outpatient department and, although the haematuria continued intermittently, he remained generally well and further IVP and cystoscopy were again judged to be normal. Three years later he had a total laryngectomy for carcinoma larynx, during which time he was unfortunately lost to follow-up. One year later he returned to our clinic because of recurrent haematuria, at which time intravenous pyelogram revealed a space-occupying lesion in the right kidney, confirmed on renal angiography as a hypernephroma. Chest X-ray showed pulmonary metastases and the patient later died.

(9) One patient in the series presented with a short history of haematuria, but no diagnosis was made after full laboratory investigation, intravenous pyelography and bilateral retrograde pyelograms. A further IVP 3 months later revealed a filling defect in the right kidney, confirmed on retrograde pyelograms. Biopsy of the nephro-ureterectomy specimen confirmed the diagnosis of squamous cell carcinoma of the renal pelvis, which was found at operation to be invading the perirenal tissues and adjacent vertebrae.

(10) One patient in the series was found to have sickle cell trait, confirmed in the literature as a possible cause of haematuria (Goodwin, Alston and Seamans, 1960); again, the finding of positive sickle cell trait must not dissuade one from full urological investigation to exclude the presence of co-existing urinary tract lesions.

In the remaining patients, no cause, conjectured, circumstantial or certain, was found.

Two further sub-groups perhaps deserve mention.

(a) Young patients. Nine of the patients in the series were under 25 years of age. Almost half of these are still under investigation with recurrent or persisting haematuria, while the remainder had one attack only with no recurrence. In none of this group has a diagnosis been found. The youngest patient in the group was 12 years of age. Benign recurrent haematuria in children is a well documented syndrome, and leads in most paediatric centres to the diagnosis (albeit controversial) of focal nephritis. Apart from the child of 12, there were no paediatric cases in our index (the other eight cases were all over sixteen) The subject of paediatric haematuria is not further discussed here.

(b) The other group worthy of mention comprises those patients who present with a story of one isolated attack of haematuria. Again, intensive investigation of these patients yielded no positive diagnosis. Such cases are especially deserving of the categorization under review.

After investigation, all patients were followed up as outpatients, and in many cases were discharged after what was judged to be a suitable period of time, during which they remained in good health and showed negative clinical findings. They were instructed to return to the clinic should the bleeding recur; few did and in those that did, excepting the hypernephroma, no cause was found.

\section{Discussion}

Positive diagnosis revealed itself in three out of sixty-four cases of haematuria presenting to this hospital, in whom no cause was found after initial but thorough investigation. The remaining sixty-one cases entered that generally unsatisfactory group, haematuria of unknown origin. Where, then, does the clinician proceed from here? If the incident described by the patient was indeed haematuria, then it is both an alarming and an important one and merits the fullest evaluation.

It is when initial investigation, including intravenous pyelography and cystoscopy, reveals no diagnosis that one must ask the following questions.

(1) What further tests are necessary or desirable;

(2) how useful are the more refined and exotic tests likely to be, when weighed against the probability of a diagnosis, expense, patient's time and discomfort and possible side-effects; and

(3) how rigorous and prolonged should follow-up be?

Further investigation will fall into three main groups-laboratory investigation, radiology, and renal biopsy. Some of the laboratory tests are discussed below.

Sickle cell test. Goodwin et al. (1960) first reported a connection between sickle cell trait and haematuria. As a rule, unlike patients with the full-blown disease, these patients are free from pain and organ involvement and haematuria may be the only symptom. Conditions in the renal papilla such as increased osmolarity, low oxygen tension and a low pH all favour clumping of the abnormal red cells with the formation of characteristic crescents. This leads to vascular occlusion with subsequent rupture 
of the vessels and extravasation of blood into the interstitial tissue and renal tubules. Renal papillary necrosis and fibrosis can occur. Thus, any patient with negroid heritage presenting with haematuria should have this test.

Twenty-four hour urinary protein is of obvious value in renal disease, and may be of special significance in the diagnosis of more severe cases of proliferative or chronic glomerulonephritis presenting as haematuria (Hendler, Kashgarian and Hayslett, 1972).

Fibrinolytic activity. Manabe (1971) reported work from Japan, where fifteen patients suffering from haematuria of undetermined origin were investigated for fibrinolytic activity. All the patients under study were found to have increased fibrinolytic activity in their blood and a higher level of fibrinolytic activator in their urine, while the controls showed an inactive fibrinolytic system. It is postulated that the affected kidney may discharge fibrinolysin activator and proactivator into the bloodstream. The significance of this finding has yet to be elucidated.

With regard to radiological procedures, the patient will already have had an intravenous pyelogram. It cannot be over-emphasized how important is close scrutiny of these films, and follow-up, if the clinician or radiologist is not satisfied. Poor films and lack of concentration of the contrast medium may require high dose or drip infusion pyelography, this often being the case in the presence of a high blood urea. Any inconsistencies or suspicious areas on the films may necessitate retrograde pyelograms. Ultrasound examination will also be of use in distinguishing between the cystic or solid nature of renal masses (Hately and Whitaker, 1973). One of the most useful and often the most mandatory examinations is renal arteriography, especially where one suspects renal carcinoma, but also in cases of renal cysts, infarction, ischaemia, necrosis and arteriovenous fistulae. Its use as a routine in these cases may be debatable, however. In the previously mentioned review by Burkholder et al., twenty-two of the twenty-five patients in the unknown origin group had renal arteriography. One further lesion was found-a previously undiagnosed arteriovenous fistula, but the remaining 21 arteriograms were normal, from which one might deduce that the procedure was not justified as a routine. However, it should be added that these workers were dealing with a group of young subjects, whose average age was 27 years. Perhaps more convincing evidence for renal arteriography might be found in the older age groups with haematuria, and particularly those with doubtful areas on their IVPs, for it is in these patients that the small hypernephroma may be missed until IVP changes are gross and the tumour widespread. This was well illustrated in the case of hyper- nephroma in the UHSM group whose neoplasm might possibly have been visualized on an arteriogram, where it was not on an intravenous pyelogram. This patient was also lost to follow-up for 3 vital years until, when he was seen again, it was too late. Full use should be made of arteriography if there is the slightest suspicion of a correctable or life-endangering lesion.

The technique of percutaneous needle biopsy of the kidney is now well established, but the interpretation of the results is not, and it may be difficult to differentiate minimal changes from normal appearances. In cases of unexplained haematuria the procedure has its opponents and proponents. In Burkholder's group, eighteen patients had needle biopsy and one an open biopsy; ten patients were said to have lesions which could have caused the haematuria, but the workers felt that the procedure was not justified as a routine in these patienst, especially since no lesion was found which resulted in any change of the management or therapy. While not justified as a routine, it is of obvious value in particular cases, such as those with significant proteinuria.

Papers have appeared claiming that patients with unexplained haematuria will in most cases have $a^{2}$ focal nephritis (Ferris et al., 1967; Ross, 1960) but it is important that focal nephritis is not regarded as a? clinical diagnosis. Brodwall, Oyri and Oystese (1971), reviewing thirty-one cases of focal nephritis, diag-? nosed on renal biopsy, found that nineteen were indeed referred because of benign recurrent haematuria. However, of the remaining twelve, seven had systemic lupus erythematosus, two nephrotic syndrome, one Schönlein-Henoch syndrome, one sarcoidosis and one myocarditis; many of these conditions can themselves be responsible for haematuria, and to label haematuria of unknown origin as focal nephritis without further investigation may be misleading.

As was previously stated, the majority of patients in the UHSM group were discharged after what was judged to be a sufficient follow-up. Yet, just one case such as the hypernephroma reminds one of how carefully controlled follow-up must be. For the purposes of their investigation, the Texas patients had almost every conceivable urological investigation, yielding but little additional information, and certainly none that resulted in any significant change in therapy.

The happy medium is probably the best management. Thorough initial investigation including laboratory investigation, cystoscopy (and cystoscopy at the time of bleeding may be of value), intravenous pyelography and, if necessary, retrograde pyelography and renal angiograms, are essential. Only then can the patient be put into the 
unknown origin category: and here, perhaps, one begins the most important part of one's management-that of follow-up. The cases at this stage are more accurately termed haematuria of unknown origin as yet. Continued observation by the family doctor rather than the hospital may well be necessary for economic and temporal reasons, but the importance of careful follow-up must be emphasized to all concerned. Without, at the very least, further mid-stream specimens of urine microscopic haematuria may persist without further investigation. In those cases with persisting haematuria it may be necessary to repeat cystoscopy, IVP and renal arteriography at a suitable interval and to perform renal biopsy to elucidate the underlying disease process; if the small, but important, group of patients with undiagnosed but significant disease can be detected and treated, then the clinician's perseverance in following up his cases of haematuria will be well rewarded.

\section{Conclusions}

In a significant number of patients referred to hospital because of haematuria, no cause was found. In our present state of knowledge, it seems likely that such haematuria of unknown origin will remain a somewhat large group in any urological disease index. Extensive investigation is unlikely to be rewarded with a diagnosis, but thorough initial investigation and careful follow-up are essential if the early hypernephroma or latent underlying disease is not to be missed, and there is no place for glib expectant treatment-by definition, haematuria of unknown origin presupposes comprehensive investigation. Only then can the patient be given his full and deserved reassurance.

\section{Acknowledgments}

My thanks are due to $\mathrm{Mr}$ A. Ashworth and Mr R. J. Barnard for permission to review the cases under their care, and for their encouragement.

\section{References}

BrodWell, E.K., OYRI, A. \& OYSTESE,'B. (1971) Intermittent benign renal haematuria. Acta medica scandinavica, 190, 545.

Burkholder, G.V., Dotin, L.N., Thomason, W. \& Beach, P.D. (1969) Unexplained haematuria. Journal of the American Medical Association, 210, 1729.

Ferris, T.F., Gorden, P., Kashgarian, M. \& Epstein, F.H. (1967) Recurrent haematuria and focal nephritis. New England Journal of Medicine, 276, 770.

Goodwin, W.G., Alston, E.F. \& Seamans, J.H. (1960) Haematuria and sickle cell disease: unexplained gross unilateral renal haematuria in negroes coincident with blood sickling trait. Journal of Urology, 63, 79.

HATEleY, W. \& WhitakeR, R.H. (1973) How accurate is diagnostic ultrasound in renal disease? British Journal of Urology, 45, 468.

Hendler, E.D., Kashgarian, M. \& Hayslett, J. (1972) Clinico-pathological correlations of primary haematuria. Lancet, $\mathbf{i}, 458$.

Manabe, H. (1971) So called essential renal haematuria and the fibrinolytic enzyme system. Acta Urologica Japonica, $17,314$.

Ross, J.H. (1960) Recurrent focal nephritis. Quarterly Journal of Medicine, 29, 391. 